\title{
SIMPLE ZEROS OF THE RIEMANN ZETA-FUNCTION
}

\author{
A. Y. CHEER AND D. A. GOLDSTON
}

(Communicated by William Adams)

\begin{abstract}
Assuming the Riemann Hypothesis, Montgomery and Taylor showed that at least $67.25 \%$ of the zeros of the Riemann zeta-function are simple. Using Montgomery and Taylor's argument together with an elementary combinatorial argument, we prove that assuming the Riemann Hypothesis at least $67.275 \%$ of the zeros are simple.
\end{abstract}

\section{INTRODUCTION}

Let $N(T)$ denote the number of zeros of the Riemann zeta-function $\zeta(s)$, $s=\sigma+i t$, in the rectangle $0<\sigma<1,0<t \leq T$. Let $N_{s}(T)$ denote the number of simple zeros of $\zeta(s)$ in the same rectangle. It is generally believed that $N_{s}(T)=N(T)$, that is, all the zeros of $\zeta(s)$ are simple. In support of this conjecture it has been shown by van de Lune, te Riele, and Winter [7] that the first $1.5 \times 10^{9}$ zeros in the critical strip are simple and lie on $\sigma=\frac{1}{2}$. Further, Conrey [2] has shown that at least $40 \%$ of the zeros of $\zeta(s)$ are simple and lie on $\sigma=\frac{1}{2}$. By $40 \%$ we mean that $N_{s}(T) / N(T)>.4$ for all sufficiently large $T$.

Assuming the Riemann Hypothesis ( $\mathrm{RH})$, Montgomery [8] proved that, for any $\varepsilon>0$, more than $\frac{2}{3}-\varepsilon$ of the zeros are simple. Montgomery and Taylor optimized the argument [9] to show that more than $\frac{3}{2}-2^{-1 / 2} \cot \left(2^{-1 / 2}\right)-\varepsilon=$ $.67250 \ldots$ of the zeros are simple. More recently, Conrey, Ghosh, and Gonek $[1,4]$ found a new argument that shows that more than $19 / 27-\varepsilon=.70370 \ldots$ of the zeros are simple; however, at present this result requires the assumption of the Riemann Hypothesis and also either the Lindelöf Hypothesis for Dirichlet L-functions or an unproved sixth power moment for L-functions on the half line.

In this paper we show that it is possible to obtain a small improvement in Montgomery and Taylor's lower bound for simple zeros.

Theorem. Assuming the Riemann Hypothesis, we have $N_{s}(T)>.67275 N(T)$ for all sufficiently large $T$.

Received by the editors February 14, 1991 and, in revised form, October 10, 1991.

1991 Mathematics Subject Classification. Primary 11M26.

The first author's research was supported in part by The Institute for Theoretical Dynamics, University of California at Davis.

The second author's research was supported in part by NSF Grant DMS 9003329. 
This result is obtained by using Montgomery and Taylor's method together with an elementary combinatorial argument. No additional information about zeros of the zeta function is used. There is also a second method that can be used to obtain an improvement in Montgomery and Taylor's result, but this method requires more information on zeros. In [3] it was shown that assuming RH there exists a positive proportion of consecutive distinct zeros whose differences are less than .77 times the average spacing. This result together with the method described in the next section leads immediately to an improvement in Montgomery and Taylor's result. However, the results in [3] depend on the same circle of ideas as used by Conrey, Ghosh, and Gonek in their work on simple zeros, although in this case the proof is much simpler. We do not develop this second method here.

The main conclusion to be drawn from this paper is that Montgomery's theorem on $F(\alpha)$ (see next section) has not yet been fully exploited, and it is an open question in harmonic analysis to determine the best result on simple zeros attainable from Montgomery's theorem.

We would like to thank Professors J. B. Conrey and A. Ghosh for helpful discussions. The second author would also like to thank the Institute for Advanced Study where some of this work was completed.

\section{MONTGOMERY AND TAYLOR'S METHOD}

Let $L=(1 / 2 \pi) \log T$. The Riemann-von Mangoldt formula states that

$$
N(T) \sim T L, \quad \text { as } T \rightarrow \infty .
$$

Assuming the Riemann Hypothesis, a typical zero of the Riemann zeta-function may be denoted $\rho=\frac{1}{2}+i \gamma$ and the multiplicity denoted by $m_{\rho}$. Define

$$
N^{*}(T)=\sum_{0<\gamma \leq T} m_{\rho},
$$

where the sum is over all zeros counting multiplicity. We can obtain a lower bound for $N_{s}(T)$ from an upper bound for $N^{*}(T)$ since

$$
N_{s}(T) \geq \sum_{0<\gamma \leq T}\left(2-m_{\rho}\right)=2 N(T)-N^{*}(T) .
$$

To obtain an upper bound for $N^{*}(T)$, we begin by defining Montgomery's function

$$
F(\alpha)=F(\alpha, T)=\frac{1}{T L} \sum_{0<\gamma, \gamma^{\prime} \leq T} e\left(\alpha\left(\gamma-\gamma^{\prime}\right) L\right) w\left(\gamma-\gamma^{\prime}\right),
$$

for $T \geq 2$, where $e(u)=e^{2 \pi i u}$ and $w(u)=4 /\left(4+u^{2}\right)$. The main information about $F(\alpha)$ is contained in the following theorem [8].

Theorem (Montgomery). The function $F(\alpha)$ is real, even, and nonnegative. Assuming the Riemann Hypothesis, we have

$$
F(\alpha)=T^{-2 \alpha} \log T(1+o(1))+\alpha+o(1), \quad \text { as } T \rightarrow \infty,
$$

uniformly for $0 \leq \alpha \leq 1$.

\footnotetext{
${ }^{1}$ Actually Montgomery proved a slightly weaker result. Mueller and Heath-Brown independently noted that $F(\alpha)$ was actually nonnegative.
} 
Montgomery conjectured that $F(\alpha)=1+o(1)$, as $T \rightarrow \infty$ uniformly for $\alpha>1$ in bounded intervals. This conjecture together with the theorem has two important consequences:

$$
N(T, \beta)=\sum_{\substack{0<\gamma, \gamma^{\prime} \leq T \\ 0<\gamma-\gamma^{\prime} \leq \beta / L}} 1 \sim T L \int_{0}^{\beta} 1-\left(\frac{\sin \pi u}{\pi u}\right)^{2} d u
$$

and

$$
N^{*}(T) \sim T L .
$$

The result in equation (6) is the pair correlation conjecture, which has received strong numerical verification by Odlyzko [10, 11]. Equation (7) implies by (1) and (3) that almost all the zeros of the zeta function are simple (asymptotically $100 \%)$. Unfortunately, Montgomery's conjecture appears difficult to prove, particularly since on RH the conjecture has been shown to be equivalent to a second moment asymptotic formula for primes in short intervals [6]. Therefore, if we only assume RH we are forced at present to replace (6) and (7) with partial results obtained from Montgomery's theorem alone.

Let $r(u) \in L^{1}$ and define the Fourier transform by

$$
\hat{r}(\alpha)=\int_{-\infty}^{\infty} r(u) e(-\alpha u) d u .
$$

If $\hat{r}(\alpha)$ is also $L^{1}$, we have almost everywhere the inversion formula

$$
r(u)=\int_{-\infty}^{\infty} \hat{r}(\alpha) e(u \alpha) d \alpha .
$$

On multipling equation (4) by $\hat{r}(\alpha)$ and integrating, we obtain

$$
\sum_{0<\gamma, \gamma^{\prime} \leq T} r\left(\left(\gamma-\gamma^{\prime}\right) L\right) w\left(\gamma-\gamma^{\prime}\right)=T L \int_{-\infty}^{\infty} \hat{r}(\alpha) F(\alpha) d \alpha .
$$

To apply the theorem we now assume $\hat{r}(\alpha)$ is even and has support in $[-1,1]$. We thus obtain

$$
\begin{gathered}
r(0) N^{*}(T)+2 \sum_{\substack{0<\gamma, \gamma^{\prime} \leq T \\
0<\gamma-\gamma^{\prime}}} r\left(\left(\gamma-\gamma^{\prime}\right) L\right) w\left(\gamma-\gamma^{\prime}\right) \\
=\left\{\hat{r}(0)+2 \int_{0}^{1} \alpha \hat{r}(\alpha) d \alpha+o(1)\right\} T L .
\end{gathered}
$$

If we now assume $r(u) \geq 0$, then the sum over pairs of zeros $0<\gamma-\gamma^{\prime}$ is nonnegative, and we obtain

$$
N^{*}(T) \leq\left\{\frac{\hat{r}(0)+2 \int_{0}^{1} \alpha \hat{r}(\alpha) d \alpha+o(1)}{r(0)}\right\} T L .
$$

The functions $k(u)=\left(\frac{\sin \pi u}{\pi u}\right)^{2}$ and $\hat{k}(\alpha)=\max (1-|\alpha|, 0)$ have the required properties used above and when substituted into (10) give $N^{*}(T) \leq$ $\left(\frac{4}{3}+o(1)\right) T L$, which implies by (1) and (3) $N_{s}(T) \geq\left(\frac{2}{3}-o(1)\right) T L$. 
To optimize the above argument, we need to minimize the right-hand side of (10), where the minimum is taken over the class of all $L^{1}$ functions that are nonnegative, even, and have Fourier transforms with support in $[-1,1]$. Montgomery and Taylor solved this problem using a variational argument [9], and Gallagher [5] has treated a slightly more general problem. Without loss of generality we may assume $\hat{r}(\alpha)=h(\alpha) * h(-\alpha)$, where $h(\alpha)$ is $L^{1}$ and has support in $\left[-\frac{1}{2}, \frac{1}{2}\right]$. The extremal function for this problem is any constant times $h(\alpha)=\cos (\sqrt{2} \alpha)$ for $|\alpha| \leq \frac{1}{2}$ and zero elsewhere. Normalizing the final extremal function to be 1 at the origin, we obtain

$$
g(u)=\frac{1}{1-\cos (\sqrt{2})}\left(\frac{\sin \left(\frac{1}{2}(\sqrt{2}-2 \pi u)\right)}{\sqrt{2}-2 \pi u}+\frac{\sin \left(\frac{1}{2}(\sqrt{2}+2 \pi u)\right)}{\sqrt{2}+2 \pi u}\right)^{2}
$$

and

$$
\hat{g}(\alpha)= \begin{cases}\frac{1}{1-\cos (\sqrt{2})}\left(\frac{1}{2 \sqrt{2}} \sin (\sqrt{2}(1-|\alpha|))+\frac{1}{2}(1-|\alpha|) \cos (\sqrt{2} \alpha)\right) & \text { if }|\alpha| \leq 1, \\ 0 \text { otherwise. } & \end{cases}
$$

Using (12) in (10), we obtain

$$
N^{*}(T) \leq\left(\frac{1}{2}+2^{-1 / 2} \cot \left(2^{-1 / 2}\right)+o(1)\right) T L .
$$

In Figure 1 we compare the graphs of the functions $k(u)$ and $g(u)$ and their transforms.

In examining the above argument, the only loss occurs in discarding the sum over pairs of zeros in going from (9) to (10). If we could prove that

$$
\sum_{\substack{0<\gamma, \gamma^{\prime} \leq T \\ 0<\gamma-\gamma^{\prime}}} r\left(\left(\gamma-\gamma^{\prime}\right) L\right) w\left(\gamma-\gamma^{\prime}\right) \geq c T L
$$

then we could replace $(10)$ with

$$
N^{*}(T) \leq\left\{\frac{\hat{r}(0)+2 \int_{0}^{1} \alpha \hat{r}(\alpha) d \alpha-2 c+o(1)}{r(0)}\right\} T L
$$
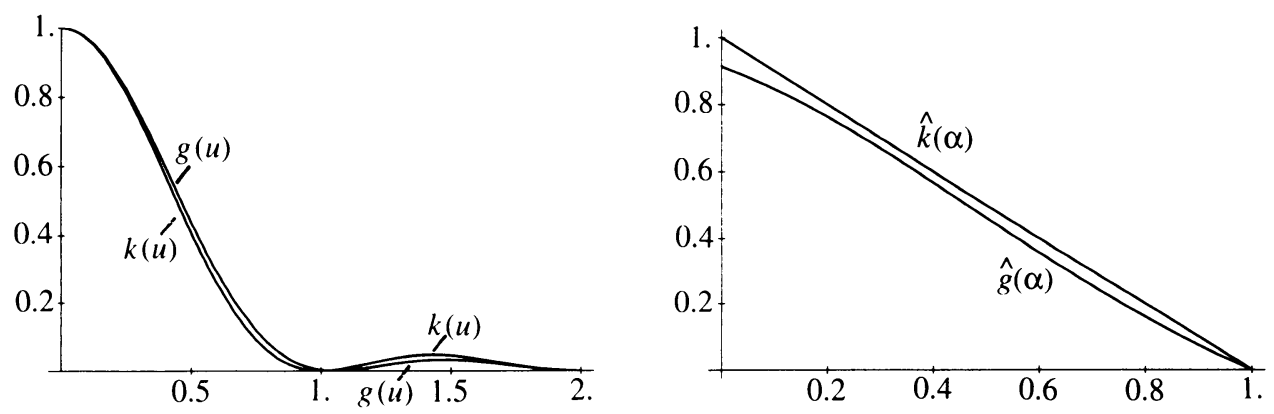

FIGURE 1. $k(u)$ and $g(u)$ (left) and $\hat{k}(\alpha)$ and $\hat{g}(\alpha)$ (right). 
To improve on Montgomery and Taylor's bound for $N^{*}(T)$, we consider (14) with $r(u)$ taken to be the function $g(u)$ in (11). The function $g(u)$ is even and has positive zeros at $\lambda_{k}, k=1,2,3, \ldots$. The values $\lambda_{k}$ are the roots of the equation $\tan (\pi u)=\tan \left(2^{-1 / 2}\right) /\left(2^{1 / 2} \pi u\right)$ for $u>\frac{1}{2}$. We see that $1<\lambda_{1}<2<\lambda_{2}<3<\lambda_{3}<4<\lambda_{4}<\cdots$, and that $\lambda_{k}-k \downarrow 0$. The first few roots are $\lambda_{1}=1.05727 \ldots, \lambda_{2}=2.03006 \ldots, \lambda_{3}=3.02024 \ldots$, $\lambda_{4}=4.01523 \ldots$, and $\lambda_{5}=5.01220 \ldots$. We need to prove there are normalized differences $\left(\gamma-\gamma^{\prime}\right) L$ away from these values. The idea we use is based on the fact that the numbers $\left(\gamma-\gamma^{\prime}\right) L$ are differences. Consider the set of consecutive differences between distinct zeros. By Montgomery's Theorem it may be shown that there are at least $\frac{5}{6} T L$ such differences, and hence the average length of these differences is at most $\frac{6}{5} T L$. If all of these differences are of length $\lambda_{1}, \lambda_{2}, \lambda_{3}, \ldots$, then there must be at least about $\frac{2}{3} T L$ differences of length $\lambda_{1}$. From this we see that there is a positive proportion of next to consecutive differences of length $2 \lambda_{1}$. Since $2 \lambda_{1} \neq \lambda_{2}$, we have produced some differences whose lengths are not at the zeros of $g(u)$. The idea of making use of the property that the set $\left\{\lambda_{k}\right\}$ is not formed from multiplies of $\lambda_{1}$ was suggested to us by J. B. Conrey.

\section{Proof of the Theorem}

Let $\left\{\lambda_{k}\right\}$ denote the sequence of positive zeros of $g(u)$ defined at the end of the last section. Let $\left\{\gamma_{d}\right\}$ denote the sequence of imaginary parts of the distinct zeros of $\zeta(s)$, and let

$$
N_{d}(T)=\sum_{0<\gamma_{d} \leq T} 1=\sum_{0<\gamma \leq T} \frac{1}{m_{\rho}}
$$

be the counting function for distinct zeros. We can obtain a lower bound for $N_{d}(T)$ from an upper bound for $N^{*}(T)$ by the following argument of Ghosh. Since

$$
2 N_{s}(T) \leq \sum_{0<\gamma \leq T} \frac{\left(m_{\rho}-2\right)\left(m_{\rho}-3\right)}{m_{\rho}}=N^{*}(T)-5 N(T)+6 N_{d}(T),
$$

we obtain by (1) and (3)

$$
N_{d}(T) \geq \frac{5 N(T)-N^{*}(T)+2 N_{s}(T)}{6} \geq \frac{3}{2} T L-\frac{1}{2} N^{*}(T)-o(T L) .
$$

We assume the Riemann Hypothesis so that the zeros lie on a line and consider the consecutive differences between distinct zeros. Let $\gamma_{d}{ }^{+}$denote the next distinct zero greater than $\gamma_{d}$. Let

$$
\mathscr{N}_{d}(T, u)=\sum_{\substack{0<\gamma_{d} \leq T \\ \gamma_{d}-\gamma_{d} \leq u / L}} 1,
$$

count the number of consecutive differences between distinct zeros of length $\leq u / L$. 


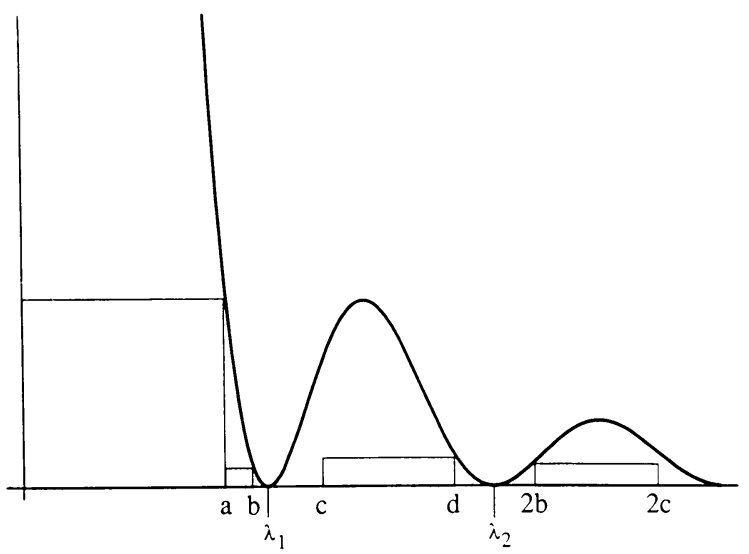

FiguRE 2. Lower rectangles for $g(u)$.

Now consider Figure 2 and suppose $0<a<b<\lambda_{1}<c<d<\lambda_{2}$ and $\lambda_{2}<2 b<2 c<\lambda_{3}$. We define the number of consecutive differences between distinct zeros with lengths in the intervals in Figure 2 by

$$
\begin{gathered}
A=\mathscr{N}_{d}(T, a), \quad B=\mathcal{N}_{d}(T, b)-\mathcal{N}_{d}(T, a), \\
N=\mathscr{N}_{d}(T, c)-\mathscr{N}_{d}(T, b), \quad C=\mathscr{N}_{d}(T, d)-\mathscr{N}_{d}(T, c), \\
R=N_{d}(T)-\mathscr{N}_{d}(T, d) .
\end{gathered}
$$

Clearly

$$
A+B+N+C+R=N_{d}(T)
$$

and since the sum of the lengths of all the consecutive differences is $\sim T$ by (1), we have

$$
a B+b N+c C+d R \leq(1+o(1)) T L .
$$

Using (19) we eliminate $R$ in equation (20) and solve for $N$ to obtain

$$
N \geq \frac{d N_{d}(T)-(1+o(1)) T L-d A-(d-a) B-(d-c) C}{d-b} .
$$

We now define $N_{2}=N_{2}(b, c)$ to be the number of next to consecutive gaps of distinct zeros of length $l / L$, with $2 b \leq l \leq 2 c$. Clearly $N_{2}$ is greater than or equal to the number of pairs of abutting distinct gaps counted by $N$ (each pair counted once), and hence by a simple combinatorial argument, we obtain

$$
N_{2} \geq \max \left\{2 N-N_{d}(T)-1,0\right\} .
$$

By equation (21) we conclude

$$
N_{2} \geq 2\left\{\frac{\frac{b+d}{2} N_{d}(T)-(1+o(1)) T L-d A-(d-a) B-(d-c) C}{d-b}\right\} .
$$


We now complete the proof of the theorem. By Figure 2, letting $h=$ $\min (g(c), g(d))$ and $j=\min (g(2 b), g(2 c))$, we have

$$
\begin{aligned}
& \sum_{\substack{0<\gamma, \gamma^{\prime} \leq T \\
0<\gamma-\gamma^{\prime}}} g\left(\left(\gamma-\gamma^{\prime}\right) L\right) w\left(\gamma-\gamma^{\prime}\right) \geq g(a) A+g(b) B+h C+j N_{2}-o(T L) \\
& \quad \geq\left(g(a)-\frac{2 j d}{d-b}\right) A+\left(g(b)-\frac{2 j(d-a)}{d-b}\right) B+\left(h-\frac{2 j(d-c)}{d-b}\right) C \\
& \quad+\frac{2 j}{d-b}\left(\frac{d+b}{2} N_{d}(T)-T L\right)-o(T L) .
\end{aligned}
$$

We choose $a, b, c$, and $d$ so that the coefficients of $A, B$, and $C$ are positive in (23), which implies since $A \geq 0, B \geq 0$, and $C \geq 0$ that

$$
\sum_{\substack{0<\gamma, \gamma^{\prime} \leq T \\ 0<\gamma-\gamma^{\prime}}} g\left(\left(\gamma-\gamma^{\prime}\right) L\right) w\left(\gamma-\gamma^{\prime}\right) \geq \frac{2 j}{d-b}\left(\frac{d+b}{2} N_{d}(T)-T L\right)-o(T L) .
$$

It is easy to maximize numerically the right-hand side of (24) by choosing $b$ as large as possible in the interval $\lambda_{2} / 2<b<\lambda_{1}$ while satisfying the three constraints. We find the choice $a=1.0233, b=1.033396, c=1.47$, $d=1.99$, with $N_{d}(T) \geq .83625 T L$ (using Montgomery's bound for $N^{*}(T)$ in (16)) gives a lower bound in (24) of $0.00012636 T L$, which by (14) gives $N^{*}(T) \leq(1.327499296 \ldots-2(0.00012636)) T L<1.3272466 T L$. The theorem now follows from (3).

\section{FinAl COMMENTS}

The function $F(\alpha)$ is real and even because $\gamma$ and $\gamma^{\prime}$ can be switched, and $F(\alpha) \geq 0$ follows immediately from the easily verified identities

$$
\begin{aligned}
F(\alpha) & =\frac{2}{\pi T L} \int_{-\infty}^{\infty}\left|\sum_{0<\gamma \leq T} \frac{e(\alpha \gamma L)}{1+(t-\gamma)^{2}}\right|^{2} d t \\
& =\frac{1}{T L} \int_{-\infty}^{\infty}\left|\sum_{0<\gamma \leq T} e(\gamma(\alpha L+t / 2 \pi))\right|^{2} e^{-2|t|} d t
\end{aligned}
$$

Thus the properties of $F(\alpha)$ being real, even, and nonnegative do not depend on $\gamma$ being the imaginary part of a zero of the zeta function, since (25) will hold for the $\gamma$ 's being any sequence of real numbers. We see that only equation (5) in Montgomery's Theorem tells us anything about zeros of the zeta function. We can therefore ask the following question of harmonic analysis: Given any sequence of real numbers $\gamma$ that satisfy (1) and the associated function $F(\alpha)$ given in (4) satisfying (5), what is the maximum of $N^{*}(T)$ defined in (2) for all sufficiently large $T$ ?

Suppose we drop equation (1), replace in equation (4) the expression $\left(\gamma-\gamma^{\prime}\right) L$ by any set of real frequencies $\nu$, and assume Montgomery's Theorem holds for this function $F(\alpha)$. Then we can ask the question: what is the maximum of $N^{*}(T)=\#\{\nu=0\}$ ?

Through numerical work it seems very likely that

$$
\frac{N^{*}(T)}{T L} \sim \frac{1}{2}+2^{-1 / 2} \cot \left(2^{-1 / 2}\right)=1.327499 \ldots
$$


is asymptotically attained in this case and $g(u)$ is the optimal kernel. Thus any improvement over this result most likely depends on using additional properties of the frequencies $\nu$. The improvement we obtained depends on using (1) and that the frequencies $\nu=\left(\gamma-\gamma^{\prime}\right) L$ are differences, but our argument is not optimal. Thus it remains an open problem to determine the best result on simple zeros that follows from Montgomery's Theorem.

\section{REFERENCES}

1. J. B. Conrey, On the distribution of the zeros of the Riemann zeta-function, Topics in Analytic Number Theory, University of Texas Press, Austin, TX, 1985, pp. 28-41.

2. - More than two-fifths of the zeros of the Riemann zeta function are on the critical line, J. Reine Angew. Math. 399 (1989), 1-26.

3. J. B. Conrey, A. Ghosh, D. Goldston, S. M. Gonek, and D. R. Heath-Brown, Distribution of gaps between zeros of the zeta function, Quart. J. Math. Oxford Ser. (2) 36 (1985), 43-51.

4. J. B. Conrey, A. Ghosh, and S. M. Gonek, Mean values of the Riemann zeta-function, Number Theory, Trace Formulas and Discrete Groups (Symposium in Honor of Atle Selberg, Oslo, Norway, July 14-21, 1987), Academic Press, New York, 1989, pp. 185-199.

5. P. X. Gallagher, Pair correlation of zeros of the zeta function, J. Reine Angew. Math. $\mathbf{3 6 2}$ (1985), 72-86.

6. D. A. Goldston and H. L. Montgomery, Pair correlation of zeros and primes in short intervals, Analytic Number Theory and Diophantine Problems, Birkhaüser, Boston, MA, 1987, pp. 183-203.

7. J. van de Lune, H. J. J. te Riele, and D. T. Winter, On the zeros of the Riemann zeta function in the critical strip. IV, Math. Comp. 46 (1986), 667-681.

8. H. L. Montgomery, The pair correlation of zeros of the zeta function, Proc. Sympos. Pure Math., vol. 24, Amer. Math. Soc., Providence, RI, 1973, pp. 181-193.

9. _- Distribution of zeros of the Riemann zeta function, Proc. Internat. Congr. Math. Vancouver, 1974, pp. 379-381.

10. A. M. Odlyzko, On the distribution of spacings between zeros of the zeta function, Math. Comp. 48 (1987), 273-308.

11. _ The $10^{20}-$ th zero of the Riemann zeta function and 70 million of its neighbors (to appear).

Department of Mathematics and Institute of Theoretical Dynamics, University of California, Davis, California 95616

E-mail address: aycheer@ucdavis.edu

Department of Mathematics and Computer Science, San Jose State University, San Jose, CALIFORNIA 95192

E-mail address: goldston@sjsumcs.sjsu.edu 